\title{
A remark concerning random walks with random potentials
}

\author{
by
}

Yakov G. S in a i (Princeton, N.J., and Moscow)

\begin{abstract}
We consider random walks where each path is equipped with a random weight which is stationary and independent in space and time. We show that under some assumptions the arising probability distributions are in a sense uniformly absolutely continuous with respect to the usual probability distribution for symmetric random walks.
\end{abstract}

We consider random walks on the $d$-dimensional lattice $\mathbb{Z}^{d}$ with each path having a random statistical weight. Paths starting at $(x, k)$ and ending at $(y, n)$ will be denoted by $\omega_{x, k}^{y, n}$, i.e. $\omega_{x, k}^{y, n}=\left\{\omega(t) \in \mathbb{Z}^{d}, k \leq t \leq n\right.$, $\omega(k)=x, \omega(n)=y,\|\omega(t+1)-\omega(t)\|=1\}$. To define a random weight introduce a sequence of iid $\mathrm{rv} F=\{F(x, t)\}, x \in \mathbb{Z}^{d}, t \in \mathbb{Z}$. Without any loss of generality we may assume that the $F(x, t)$ are given for all $x \in \mathbb{Z}^{d}, t \in \mathbb{Z}$. The space of all possible realizations of $F$ is denoted by $\Phi$. The measure corresponding to $F$ is denoted by $Q$, the expectation with respect to $Q$ is denoted by $M$. We do not use any special notation for the natural $\sigma$-algebra in $\Phi$. Our main assumption concerning the distribution of $F(x, t)$ is

$$
M \exp (2 F(x, t))<\infty .
$$

The natural group of space-time translations acting in $\Phi$ is denoted by $\left\{T^{x, t}\right\}$. It preserves the measure $Q$.

We shall consider the statistical weight of $\omega_{x, k}^{y, n}$ equal to

$$
\pi\left(\omega_{x, k}^{y, n}\right)=\exp \left\{\sum_{t=k}^{n} F(t, \omega(t))\right\} \frac{1}{(2 d)^{n-k}} .
$$

Introduce partition functions

$$
Z_{x, k}^{y, n}=\sum_{\omega_{x, k}^{y, n}} \pi\left(\omega_{x, k}^{y, n}\right), \quad Z_{x, k}^{n}=\sum_{y} Z_{x, k}^{y, n}
$$

1991 Mathematics Subject Classification: Primary 34F05. 
Now we may define the "random" probability distribution $P_{F ; x, k}^{n}$ defined on paths $\omega_{x, k}^{y, n}$ by the formula

$$
p\left(\omega_{x, k}^{y, n}\right)=\frac{\pi\left(\omega_{x, k}^{y, n}\right)}{Z_{x, k}^{n}} .
$$

The induced probability distribution of $y=\omega(n)$ is

$$
p_{x, k}^{y, n}=\sum_{\omega_{x, k}^{y, n}} p\left(\omega_{x, k}^{y, n}\right)=\frac{Z_{x, k}^{y, n}}{Z_{x, k}^{n}} .
$$

We shall also need the usual transition probabilities

$$
q^{(n-k)}(y-x)=\sum_{\substack{\omega_{x, k}^{y, n} \\ x}} \frac{1}{(2 d)^{n-k}} .
$$

It is well known that for any $A>0$ and $y$ for which $\|y-x\| \leq A \sqrt{n}$,

$$
\begin{aligned}
q^{(n-k)}(y-x) & 1 \\
& =\frac{1}{(2 \pi(n-k) / d)^{d / 2}} \exp \left\{-\frac{d\|y-x\|^{2}}{2(n-k)}\right\}\left(1+\gamma^{(n-k)}(y-x)\right),
\end{aligned}
$$

where $\|\cdot\|$ is the Euclidean norm and $\gamma^{(n-k)}(z)$ tends to zero uniformly in $z$ satisfying the above-mentioned restrictions.

Our purpose in this note is to study the behavior of the distribution of the normalized displacement

$$
\frac{\omega(n)-\omega(k)}{\sqrt{(n-k) / d}}=\frac{y-x}{\sqrt{(n-k) / d}}
$$

with respect to $P_{F ; x, k}^{n}$ as $n \rightarrow \infty$. The problem was considered by J. Imbrie and T. Spencer [3] and later by E. Bolthausen [1]. In [1] and [3], it was shown that if the $F(x, t)$ are small enough in appropriate sense, and $d \geq 3$, then the limiting distribution of the displacement is Gaussian and for typical $F$ the mean of the square of displacement grows proportionally to time. Recently these results were extended to some random processes with continuous time by J. Conlon and P. Olsen [2]. All these results can be formulated also in terms of diffusion of directed polymers in random environments.

We show below that some of the results of [1] and [3] remain valid under weaker assumptions on the distribution of $F$ and the dimension $d$. Define

$$
\alpha_{d}=\sum_{n>0} \sum_{z}\left(q^{(n)}(z)\right)^{2}
$$

This is finite if $d \geq 3$. Put

$$
\Lambda=M \exp \{F(x, t)\} \quad \text { and } \quad \lambda=\frac{M \exp \{2 F(x, t)\}-\Lambda^{2}}{\Lambda^{2}} .
$$


Our main assumption is

$$
\lambda \alpha_{d}<1 .
$$

It is easy to see that (1) is valid for $d \geq 3$ if $\lambda$ is small enough. If $F(x, t)$ takes two values $\pm c$ with probability $1 / 2$, then (1) is valid for those $d$ for which $\alpha_{d}<1$, and does not require the smallness of $c$. Indeed, in this case always $\lambda<1$, i.e.,

$$
M \exp \{2 F(x, t)\} \leq 2 \Lambda^{2},
$$

because this is equivalent to the obvious inequality

$$
\frac{1}{2}\left(e^{2 c}+e^{-2 c}\right) \leq 2\left(\frac{e^{c}+e^{-c}}{2}\right)^{2} .
$$

If the $F(x, t)$ have Gaussian distribution $N(0, \sigma)$, then $(1)$ is valid for small enough $\sigma$.

Put

$$
h(x, t)=\frac{\exp \{F(x, t)\}-\Lambda}{\Lambda}
$$

and introduce the series

$$
\begin{aligned}
\varphi(x, k)= & \sum_{r \geq 1} \sum_{k \leq k_{1}<\ldots<k_{r}} \sum_{z_{1}, \ldots, z_{r}} q^{\left(k_{1}-k\right)}\left(z_{1}-x\right) q^{\left(k_{2}-k_{1}\right)}\left(z_{2}-z_{1}\right) \ldots \\
& \ldots q^{\left(k_{r}-k_{r-1}\right)}\left(z_{r}-z_{r-1}\right) h\left(z_{1}, k_{1}\right) h\left(z_{2}, k_{2}\right) \ldots h\left(z_{r}, k_{r}\right), \\
\psi(y, n)= & \sum_{r \geq 1} \sum_{k_{1}<\ldots<k_{r} \leq n} \sum_{z_{1}, \ldots, z_{r}} q^{\left(k_{2}-k_{1}\right)}\left(z_{2}-z_{1}\right) \ldots \\
& \ldots q^{\left(k_{r}-k_{r-1}\right)}\left(z_{r}-z_{r-1}\right) q^{\left(n-k_{r}\right)}\left(y-z_{r}\right) h\left(z_{1}, k_{1}\right) \ldots h\left(z_{r}, k_{r}\right) .
\end{aligned}
$$

It is clear that $\varphi(x, t)$ and $\psi(y, t)$ constitute stationary (with respect to space-time translations) random fields, i.e. $\varphi(x, k)=T^{x, k} \varphi(0,0)$ and $\psi(y, n)$ $=T^{y, n} \psi(0,0)$. Also they are transformed into each other by reversal of time in random walks. This implies, in particular, that the distributions of $\varphi(x, t)$ and $\psi(x, t)$ coincide.

Below we prove the following theorems.

THEOREM 1. If (1) is valid then the series giving $\varphi(x, k)$ and $\psi(y, n)$ converge in the space $L^{2}(\Phi, Q)$.

Theorem 2. If (1) is valid and $\|y-x\| \leq A \sqrt{n-k}$ where $A$ is any constant, then the partition function $Z_{x, k}^{y, n}$ has the representation

$$
Z_{x, k}^{y, n}=\Lambda^{n-k+1} q^{(n-k)}(y-x)\left[(1+\varphi(x, k))(1+\psi(y, n))+\delta_{(x, k)}^{(y, n)}\right],
$$

where $M \delta_{x, k}^{y, n}=0$ and $M\left(\delta_{x, k}^{y, n}\right)^{2} \rightarrow 0$ as $n \rightarrow \infty, x, k$ remain fixed and $y$ satisfies the above-mentioned restriction. 
Proof of Theorem 1. It is clear that $\varphi$ and $\psi$ are represented as sums of orthogonal vectors in the space $L^{2}(\Phi, Q)$. Therefore

$$
\begin{aligned}
M \varphi^{2}(x, k)= & \sum_{r \geq 1} \lambda^{r} \sum_{k<k_{1}<\ldots<k_{r}} \sum_{z_{1}, \ldots, z_{r}}\left(q^{\left(k_{1}-k\right)}\left(z_{1}-x\right)\right)^{2} \\
& \times\left(q^{\left(k_{2}-k_{1}\right)}\left(z_{2}-z_{1}\right)\right)^{2} \ldots\left(q^{\left(k_{r}-k_{r-1}\right)}\left(z_{r}-z_{r-1}\right)\right)^{2} \\
= & \sum_{r \geq 1}\left(\lambda \alpha_{d}\right)^{r}<\infty .
\end{aligned}
$$

The same is true for $\psi(x, t)$. We also have $M \varphi(x, k)=M \psi(y, n)=0$.

Theorem 2 is proven in Appendix 1.

THEOREM 3. If (1) holds then $1+\varphi(x, t)>0$ and $1+\psi(y, t)>0$ for Q-a.e. F.

Proof. We already showed that $M \varphi(x, t)=M \psi(x, t)=0, M \varphi^{2}(x, t)$ $>0$ and $M \psi^{2}(x, t)>0$. It is enough to consider $\varphi(x, k)$ since $\varphi(x, k)$ and $\psi(y, n)$ have the same distribution. By Theorem 2,

$$
\frac{Z_{x, k}^{y, n}}{\Lambda^{n-k+1}}-q^{(n-k)}(y-x)[(1+\varphi(x, k))(1+\psi(y, n))]=\delta_{(x, k)}^{(y, n)} q^{(n-k)}(y-x) .
$$

Take a continuous non-negative function $f$ with compact support on $\mathbb{R}^{d}$, and write

$$
\begin{aligned}
\sum_{y} \frac{Z_{x, k}^{y, n}}{\Lambda^{n-k+1}} f( & \left.\frac{x-y}{\sqrt{n-k}} \sqrt{d}\right) \\
= & (1+\varphi(x, k)) \sum_{y} q^{(n-k)}(y-x) f\left(\frac{y-x}{\sqrt{n-k}} \sqrt{d}\right)(1+\psi(y, n)) \\
& +\sum_{y} q^{(n-k)}(y-x) f\left(\frac{y-x}{\sqrt{n-k}} \sqrt{d}\right) \delta_{(x, k)}^{(y, n)} .
\end{aligned}
$$

Theorem 2 immediately implies that the last term tends to zero in $L^{2}(\Phi, Q)$ for any fixed $x, k$ and $n \rightarrow \infty$. Since $M \psi(y, n)=0$ the sum

$$
\sum_{y} q^{(n-k)}(y-x) f\left(\frac{y-x}{\sqrt{n-k}} \sqrt{d}\right)(1+\psi(y, n))
$$

converges in $L^{2}(\Phi, Q)$ to $C=\int e^{-\|z\|^{2} / 2} f(z) d z /(2 \pi)^{d / 2}>0$. Thus

$$
\lim _{n \rightarrow \infty} \frac{1}{C} \sum_{y} \frac{Z_{x, k}^{y, n}}{\Lambda^{n-k+1}} f\left(\frac{y-x}{\sqrt{n-k}} \sqrt{d}\right)=1+\varphi(x, k) \text {. }
$$


Now we can use the obvious inequality

$$
Z_{x, k-2}^{y, n} \geq \sum_{\left\langle x, x^{\prime}\right\rangle}\left(\frac{1}{2 d}\right)^{2} e^{F(x, k-2)+F\left(x^{\prime}, k-1\right)} Z_{x, k}^{y, n}=g(x, k-2) Z_{x, k}^{y, n},
$$

where the last expression gives also the definition of $g(x, k-2)$ which is positive a.e., and the sum is taken over $x^{\prime}$ such that $\left\|x-x^{\prime}\right\|=1$. We use the notation $\left\langle x, x^{\prime}\right\rangle$ for the nearest neighbors on the lattice. Thus we have

$$
1+\varphi(x, k-2) \geq g(x, k-2)(1+\varphi(x, k)) .
$$

Assume that $1+\varphi(x, k-2)=0$ with positive probability. Take $x$ and consider the set $\mathcal{H}^{+}$of those numbers $2 k$ such that $1+\varphi(x, 2 k)>0$. It follows from (2) that if $2 k \in \mathcal{H}^{+}$then $2 k-2 \in \mathcal{H}^{+}$. Therefore $\mathcal{H}^{+}=2 \mathbb{Z}^{1}$ for a.e. $F$. The ergodicity of $T^{0,2}$ implies that $Q(\{F: 1+\varphi(x, k)=0\})=0$.

Let the conditions of Theorem 2 be valid. As in the proof of Theorem 3 take a continuous function $f$ on $\mathbb{R}^{d}$ with compact support. Using Theorem 2 we can write

$$
\begin{aligned}
\sum_{y} f\left(\frac{y-x}{\sqrt{n-k}}\right. & \sqrt{d}) \frac{Z_{x, k}^{y, n}}{Z_{x, k}^{n}} \\
= & \frac{(1+\varphi(x, k)) \Lambda^{n-k+1}}{Z_{x, k}^{n}} \\
& \times\left[\sum_{y} f\left(\frac{y-x}{\sqrt{n-k}} \sqrt{d}\right) q^{(n-k)}(y-x)(1+\psi(y, n))\right. \\
& \left.+\sum_{y} f\left(\frac{y-x}{\sqrt{n-k}} \sqrt{d}\right) \delta_{(x, k)}^{(y, n)} q^{(n-k)}(y-x)\right] .
\end{aligned}
$$

Our estimations during the proof of Theorem 2 in the Appendix give

$$
\lim _{n \rightarrow \infty} \frac{Z_{x, k}^{n}}{\Lambda^{n-k+1}}=1+\varphi(x, k) .
$$

Also the last sum in (3) tends to zero in $L^{2}(\Phi, Q)$ as $n \rightarrow \infty$. Therefore, we have the following theorem.

\section{THEOREM 4.}

$$
\operatorname{lin}_{n \rightarrow \infty} \frac{1}{Z_{x, k}^{n}} \sum_{y} f\left(\frac{y-x}{\sqrt{n-k}} \sqrt{d}\right) Z_{x, k}^{y, n}=\int f(z) e^{-\|z\|^{2} / 2} \frac{d z}{(2 \pi)^{d / 2}} .
$$

This theorem shows in what sense the normalized displacement $(\omega(n)-$ $\omega(k)) \sqrt{d} / \sqrt{n-k}$ has the limiting Gaussian distribution. Its variance is the same as for the usual random walk. 


\section{Appendix}

Proof of Theorem 2. We have

$$
\begin{aligned}
Z_{x, k}^{y, n}= & \sum_{\omega_{x, k}^{y, n}} \exp \left\{\sum_{t=k}^{n} F(t, \omega(t))\right\} \frac{1}{(2 d)^{n-k}} \\
= & \sum_{\omega_{x, k}^{y, n}} \prod_{t=k}^{n}(\Lambda+\exp \{F(t, \omega(t))\}-\Lambda) \frac{1}{(2 d)^{n-k}} \\
= & \Lambda^{n-k+1} \sum_{\omega_{x, k}^{y, n}} \prod_{t=k}^{n}(1+h(\omega(t), t)) \frac{1}{(2 d)^{n-k}} \\
= & \Lambda^{n-k+1}\left[q^{(n-k)}(y-x)+\sum_{r \geq 1} \sum_{k \leq k_{1}<\ldots<k_{r}<n z_{1}, \ldots, z_{r}} q^{\left(k_{1}-k\right)}\left(z_{1}-x\right)\right. \\
& \times q^{\left(k_{2}-k_{1}\right)}\left(z_{2}-z_{1}\right) \ldots q^{\left(k_{r}-k_{r-1}\right)}\left(z_{r}-z_{r-1}\right) \\
& \left.\times q^{\left(n-k_{r}\right)}\left(y-z_{r}\right) h\left(z_{1}, k_{1}\right) \ldots h\left(z_{r}, k_{r}\right) h(y, n)\right] .
\end{aligned}
$$

In what follows we only deal with the finite sum

$$
\begin{aligned}
\widetilde{Z}_{x, k}^{y, n}= & \sum_{r \geq 1} \sum_{k \leq k_{1}<\ldots<k_{r} \leq n} \sum_{z_{1}, \ldots, z_{r}} q^{\left(k_{1}-k\right)}\left(z_{1}-x\right) q^{\left(k_{2}-k_{1}\right)}\left(z_{2}-z_{1}\right) \ldots \\
& \ldots q^{\left(k_{r}-k_{r-1}\right)}\left(z_{r}-z_{r-1}\right) q^{\left(n-k_{r}\right)}\left(y-z_{r}\right) h\left(z_{1}, k_{1}\right) \ldots h\left(z_{r}, k_{r}\right) .
\end{aligned}
$$

It is clear that $M \widetilde{Z}_{x, k}^{y, n}=0$ and

$$
\begin{aligned}
M\left(\widetilde{Z}_{x, k}^{y, n}\right)^{2}= & \sum_{r \geq 1} \lambda^{r} \sum_{\substack{k \leq k_{1}<\ldots<k_{r} \leq n \\
z_{1}, \ldots, z_{r}}}\left(q^{\left(k_{1}-k\right)}\left(z_{1}-x\right)\right)^{2} \\
& \times\left(q^{\left(k_{2}-k_{1}\right)}\left(z_{2}-z_{1}\right)\right)^{2} \ldots\left(q^{\left(n-k_{r}\right)}\left(y-z_{r}\right)\right)^{2} .
\end{aligned}
$$

Fix some constant $B$ whose value will be chosen later and consider

$$
\begin{aligned}
\widetilde{Z}_{x, k}^{y, n}(1)= & \sum_{r \leq B \ln n} \sum_{k \leq k_{1}<\ldots<k_{r} \leq n} \sum_{z_{1}, \ldots, z_{r}} q^{\left(k_{1}\right)}\left(z_{1}-x\right) \\
& \times q^{\left(k_{2}-k_{1}\right)}\left(z_{2}-z_{1}\right) \ldots q^{\left(k_{r}-k_{r-1}\right)}\left(z_{r}-z_{r-1}\right) \\
& \times q^{\left(n-k_{r}\right)}\left(y-z_{r}\right) h\left(z_{1}, k_{1}\right) \ldots h\left(z_{r}, k_{r}\right) .
\end{aligned}
$$

Let $\widetilde{Z}_{x, k}^{y, n}(2)$ be a similar sum where $r>B \ln n$. Then the trivial estimation gives

$$
M\left(\widetilde{Z}_{x, k}^{y, n}(2)\right)^{2} \leq \sum_{r>B \ln n}\left(\lambda \alpha_{d}\right)^{r}=\frac{\left(\lambda \alpha_{d}\right)^{B \ln n}}{1-\lambda \alpha_{d}}
$$


Take $B$ so large that

$$
\frac{\left(\lambda \alpha_{d}\right)^{B \ln n}}{1-\lambda \alpha_{d}} \leq \frac{1}{n^{2 d}} \quad \text { for all large enough } n .
$$

We can write

$$
\frac{Z_{x, k}^{y, n}}{\Lambda^{n-k+1}}=q^{(n-k)}(y-x)\left(1+\widetilde{Z}_{x, k}^{y, n}(1)+\widetilde{Z}_{x, k}^{y, n}(2)\right) .
$$

From our estimations it follows that

(i) for all $y$ with $\|y-x\| \leq A \sqrt{n-k}$ the ratio $\widetilde{Z}_{x, k}^{y, n}(2) / q^{(n-k)}(y-x)$ tends to zero in $L^{2}(\Phi, Q)$ uniformly in $y$;

(ii) for any continuous function $f$ with compact support, the sum

$$
\sum_{y} f\left(\frac{y-x}{\sqrt{(n-k) / d}}\right) \widetilde{Z}_{x, k}^{y, n}(2)
$$

converges to zero in $L^{2}(\Phi, Q)$.

Thus it remains to study $\widetilde{Z}_{x, k}^{y, n}(1)$ assuming $\|y-x\| \leq A \sqrt{n-k}$. Let us call an interval $\left(k_{j-1}, k_{j}\right)$ large if $k_{j}-k_{j-1} \geq n^{\beta}$ for some $\beta$ with $1 / 2<\beta<1$. Here $k_{0}=k, k_{r+1}=n$. If $r \leq B \ln n$ then at least one large interval in the sequence $\left(0, k_{1}, k_{2}, \ldots, k_{r}, n\right)$ is present. We shall show that the main contribution to $\widetilde{Z}_{x, k}^{y, n}(1)$ comes from $r$-tuples $\left(k_{1}, k_{2}, \ldots, k_{r}\right)$ with only one large interval. Write

$$
\begin{aligned}
& \widetilde{Z}_{x, k}^{y, n}(1,1) \\
& =\sum_{\substack{0 \leq r_{1} \leq B \ln n \\
0 \leq r_{2} \leq B \ln n \\
1 \leq r=r_{1}+r_{2} \leq B \ln n}} \sum_{\begin{array}{c}
\left.\boldsymbol{r}_{r_{1}}, k_{r_{1}+1}\right) \text { is the unique } \\
\text { large interval }
\end{array}} \sum_{z_{1}, \ldots, z_{r}} q^{\left(k_{1}\right)}\left(z_{1}-x\right) \\
& \quad \times q^{\left(k_{2}-k_{1}\right)}\left(z_{2}-z_{1}\right) \ldots q^{\left(k_{r_{1}+1}-k_{r_{1}}\right)}\left(z_{r_{1}+1}-z_{r_{1}}\right) \\
& \quad \times q^{\left(k_{r_{1}+2}-k_{r_{1}+1}\right)}\left(z_{r_{1}+2}-z_{r_{1}+1}\right) \ldots q^{\left(k_{r}-k_{r-1}\right)}\left(z_{r}-z_{r-1}\right) q^{\left(n-k_{r}\right)}\left(y-z_{r}\right) \\
& \quad \times\left[h\left(z_{1}, k_{1}\right) \ldots h\left(z_{r_{1}}, k_{r_{1}}\right)\right] \cdot\left[h\left(z_{r_{1}+1}, k_{r_{1}+1}\right) \ldots h\left(z_{r}, k_{r}\right)\right] .
\end{aligned}
$$

We can write

$$
\frac{\widetilde{Z}_{x, k}^{y, n}(1,1)}{q^{(n)}(y-x)}=(1+\varphi(x, k))(1+\psi(y, n))-1+\delta_{(x, k)}^{(y, n)}(2) .
$$

The last formula also implies the definition of $\delta_{(x, k)}^{(y, n)}(2)$. Since we can restrict ourselves by summation over those $\left(z_{1}, \ldots, z_{r}\right)$ where $\left\|z_{r_{1}}-x\right\| \leq n^{2 \beta}$, $\left\|z_{r_{1}+1}-y\right\| \leq n^{2 \beta}$, the summation over all other $z$ is exceedingly small. Thus $M\left(\delta_{x, k}^{y, n}(2)\right)^{2} \rightarrow 0$ as $n \rightarrow \infty$ uniformly over all $y$ under consideration. 
The rest of our argument is to show that the contribution of $r$-tuples where the number of large intervals is greater than 1 is relatively small. Again we write down the square of the norm of the corresponding sum:

$$
\begin{aligned}
S_{x, k}^{y, n}= & \alpha_{d} \sum_{r \geq 1}\left(\alpha_{d} \lambda\right)^{r} \sum_{k \leq k_{1}<\ldots<k_{r} \leq n} \sum_{z_{1}, \ldots, z_{r}} p^{\left(k_{1}-k\right)}\left(z_{1}-x\right) \\
& \times p^{\left(k_{2}-k_{1}\right)}\left(z_{2}-z_{1}\right) \ldots p^{\left(k_{r}-k_{r-1}\right)}\left(z_{2}-z_{1}\right) \ldots \\
& \ldots p^{\left(k_{r}-k_{r-1}\right)}\left(z_{r}-z_{r-1}\right) p^{\left(n-k_{r}\right)}\left(y-z_{r}\right)
\end{aligned}
$$

where $p^{(i)}(z)=\left(q^{(i)}(z)\right)^{2} / \alpha_{d}$. The last double sum can again be considered as the probability that the sum $\vec{\eta}_{1}+\ldots+\vec{\eta}_{r}$ takes the values $y-x, n-k$, where $\vec{\eta}_{j}=\left(z_{j}-z_{j-1}, k_{j}-k_{j-1}\right)$. It is easy to show that the distribution of the time component of $\eta_{j}$ decays as const $/ t^{d / 2}$. Direct probabilistic arguments show that the probability to have at least two values of $j$ for which the value of the "time" component is greater than $n^{\beta}$ decays as $1 / n^{(\beta+1) d}$. This shows that the contribution of terms with two large increments $\left(k_{j}-k_{j-1}\right)$ to $S_{x, k}^{y, n}(1)$ is small in $L^{2}(\Phi, Q)$ compared with the norm of $q^{(n-k)}(y-x)$.

We omit the details.

I thank K. M. Khanin and Yu. I. Kifer for useful discussions.

The financial support from NSF (grant DMS-9404437) and from the Russian Foundation of Fundamental Research (grant N93-01-16090) are highly appreciated.

\section{References}

[1] E. Bolthausen, A note on the diffusion of directed polymers in a random environment, Comm. Math. Phys. 123 (1989), 529-534.

[2] J. G. Conlon and P. A. Olson, A Brownian motion version of directed polymer problem, preprint, University of Michigan, 1994.

[3] J. Z. Imbrie and T. Spencer, Diffusion of directed polymers in a random environment, J. Statist. Phys. 52 (1988), 609-626. 